Variational principles in quantum statistical mechanics

This content has been downloaded from IOPscience. Please scroll down to see the full text.

1987 Eur. J. Phys. 812

(http://iopscience.iop.org/0143-0807/8/1/004)

View the table of contents for this issue, or go to the journal homepage for more

Download details:

IP Address: 193.136.215.65

This content was downloaded on 17/04/2017 at 15:59

Please note that terms and conditions apply.

You may also be interested in:

Foundations of statistical mechanics

O Penrose

Convexity inequalities for estimating free energy and relative entropy

M B Ruskai and F H Stillinger

Variational formulation of the Vlasov equation

$\mathrm{J} d a$ Providencia and C Fiolhais

Increase of Average Energy by the Process of Measurement

J. Daboul

Maximum entropy production rate in quantum thermodynamics

Gian Paolo Beretta

On the statistical mechanics of quartic type anharmonic oscillators, application of variational and perturbation methods

W Witschel and J Bohmann

Nonequilibrium thermodynamics

W A Robin

Equilibration, thermalisation, and the emergence of statistical mechanics in closed quantum systems Christian Gogolin and Jens Eisert

Thermodynamic fluctuations within the Gibbs and Einstein approaches

Yurii G Rudoi and Alexander D Sukhanov 


\title{
Variational principles in quantum statistical mechanics
}

\section{João da Providência and Carlos Fiolhais}

\author{
Departamento de Fisica, Universidade de Coimbra, P-3000 Coimbra, Portugal
}

Received 10 February 1986, in final form 29 August 1986

\begin{abstract}
The principle of least information is used to derive the inequality between the arithmetic and the geometric mean. Some important variational results in quantum statistical mechanics (Peierls and Bogolyubov inequalities) are pedagogically reviewed. Emphasis is put on the distinction between mixed states describing metastable and stable equilibrium.
\end{abstract}

\section{Introduction}

The most pursued but nevertheless still unachieved project in statistical physics has been the establishment of its compatibility with classical or quantum mechanics. The ergodic theorem, which is often invoked in this context, is not universally valid. In fact, the statement that all points of the microcanonical surface are equally accessible, is verified neither in the trivial case of a perfect gas nor for a large number of more realistic systems. Furthermore, ergodicity is not sufficient to guarantee the occurrence of irreversible phenomena, the stronger concept of 'mixing' being required.

Some authors (e.g. Katz 1967, Landsberg 1978b) therefore prefer an axiomatic approach to statistical mechanics, and find it useful to resort to the language of information theory. The equivalence of the results arising from the principle of least information with those of the Gibbs-Einstein ensemble theory does assure the connection with the known phenomenology.

We present in this article a scheme for the construction of the canonical density matrix along axiomatic lines, having in mind those students who feel uneasy with the microcanonical ensemble (we refer the reader to textbooks like Katz (1967) and Landsberg (1978b)). Realistic systems are never perfectly isolated so that it is meaningful to start from the concept of least information implied by a complex though weak external perturbation. Our construction consists in a two-step minimisation of a well defined functional of the density matrix $\hat{D}$, which is related to the information. The two steps are as follow.

(i) Minimisation with respect to the eigenvectors of
Résumé On use le principe de la moindre information pour dériver l'inéqualité entre les moyennes arithmétique et géométrique. Quelques résultats importants dans le cadre de la mécanique statistique quantique (inéqualités de Peierls et de Bogolyubov) sont révus avec un but pédagogique. On met l'accent sur la distinction entre états de mélange qui décrivent l'équilibre metastable et stable.

$\hat{\mathbf{D}}$, keeping the eigenvalues fixed. This means that we try to replace $\hat{\mathbf{D}}$ by a different matrix $\hat{\mathbf{D}}^{\prime}$ which has the same eigenvalues as $\hat{D}$ but different eigenvectors: $\hat{\mathbf{D}}|m\rangle=P_{m}|m\rangle, \hat{\mathbf{D}}^{\prime}\left|m^{\prime}\right\rangle=P_{m}\left|m^{\prime}\right\rangle,|m\rangle \neq\left|m^{\prime}\right\rangle$, We try to optimise our functional with respect to $\left\{\left|m^{\prime}\right\rangle\right\}$.

(ii) Minimisation with respect to the eigenvalues of $\hat{D}$, keeping fixed the eigenvectors previously determined. Now we replace $\hat{\mathbf{D}}$ by a matrix $\hat{\mathbf{D}}^{\prime}$ with the same eigenvectors but different eigenvalues: $\hat{\mathrm{D}}|m\rangle=P_{m}|m\rangle$, $\hat{\mathbf{D}}^{\prime}|m\rangle=P_{m}^{\prime}|m\rangle, P_{m} \neq P_{m}^{\prime}$. We try to optimise our functional with respect to $\left\{P_{m}^{\prime}\right\}$.

If minimisation (i) alone is performed and a convenient $\hat{D}$ is chosen, the inequality between the arithmetic and the geometric means is obtained.

If we compel $\hat{\mathbf{D}}$ to be diagonal in some prescribed basis and minimise our functional with respect to the eigenvalues of $\hat{\mathbf{D}}$ (step (ii)), the Peierls inequality is obtained. If the Hamiltonian is written as a sum of two terms, $\hat{\mathbf{H}}=\hat{\mathbf{H}}_{1}+\hat{\mathbf{H}}_{2}$, and the functional is evaluated for a convenient density matrix $\hat{\mathbf{D}}_{1}$, satisfying $\left[\hat{\mathbf{D}}_{1}, \hat{\mathbf{H}}_{1}\right]=$ 0 , the Bogolyubov inequality results.

Our formalism is appropriate to stress the important distinction between thermal equilibrium, which corresponds to the fulfilment of (i) and (ii), and weaker forms of equilibrium such as the kind of metastable equilibrium implied by minimisation (i) alone.

In $\S 2$, we present the two-stage minimisation of the information, in $\& 3$ we extract some particular results, and in $\$ 4$ we discuss the differences between the states, which we have asociated with stable and metastable equilibrium. The conclusions are summarised in the last section. 


\section{Least information}

As usual, we accept the following postulates:

(1) The information about the state of a complex quantal system is contained in an hermitian positivedefinite matrix $\hat{\mathbf{D}}$, which has trace one:

$$
\operatorname{Tr} \hat{\mathbf{D}}=1 \text {. }
$$

The connection with a macroscopic measurement $O$, corresponding to an observable $\mathbf{O}$, is made through the relation

$$
O=\operatorname{Tr}(\hat{\mathbf{O}} \mathbf{\mathbf { D }}) \text {. }
$$

(2) The information is represented by the functional

$$
I[\hat{\mathbf{D}}]=\operatorname{Tr}(\hat{\mathbf{D}} \log \hat{\mathbf{D}}),
$$

this choice relying on physical considerations. The state of thermal equilibrium corresponds to the least information (or maximal entropy, the entropy being defined as the symmetric of the information) compatible with imposed constraints. It is clear that no new physics is generated just by giving new names to old concepts, such as, for instance, talking about information instead of entropy. Nevertheless, we think that the word 'information' may be of some pedagogical utility.

Suppose that the energy $E=\operatorname{Tr}(\hat{\mathbf{H}} \mathbf{D})$ of a system ruled by the Hamiltonian $\hat{\mathbf{H}}$ is kept fixed. The information (2.3) should therefore be minimised with respect to all possible variations of $\hat{\mathbf{D}}$ which preserve the energy. We are going to look for the minimum of

$$
\begin{array}{r}
F[\hat{\mathbf{D}}]=I[\hat{\mathbf{D}}]+\beta E[\hat{\mathbf{D}}] \\
=\operatorname{Tr}(\hat{\mathbf{D}} \log \hat{\mathbf{D}})+\beta \operatorname{Tr}(\hat{\mathbf{D}} \hat{\mathbf{H}})
\end{array}
$$

where $\beta \in P$ is a Lagrange multiplier. It is clear that if we associate $\beta$ with the inverse absolute temperature, the functional $F[\hat{D}]$ is proportional to the Helmholtz free energy of thermodynamics.

The minimisation of (2.4) is carried out in two stages, as indicated in the introduction.

(i) Minimisation with respect to the eigenvectors of $\hat{D}$ We assume a given discrete spectrum of eigenvalues of $\hat{\mathrm{D}},\left\{P_{m} ; m=1,2, \ldots\right\}$, and minimise (2.4) within the set

$$
\mathscr{U}=\left\{\hat{\mathbf{D}}: \text { spectrum of } \hat{\mathbf{D}}=\left\{P_{m} ; m=1,2, \ldots\right\}\right. \text {. }
$$

The information (2.3) is unaffected by this process since it depends only on the eigenvalues of $\hat{\mathbf{D}}$ :

$$
I[\hat{\mathbf{D}}]=I\left[\left\{P_{m}\right\}\right]=\sum_{m} P_{m} \log P_{m}
$$

One is only faced with the task of minimising $\operatorname{Tr}(\hat{D} \hat{H})$ in $\mathscr{H}$. This problem is similar to the Ritz variational principle of quantum mechanics. A necessary condition is clearly (with $\beta \neq 0$ )

$$
\delta_{1} \operatorname{Tr}(\hat{\mathbf{D}} \hat{\mathbf{H}})=0 .
$$

The variation indicated by $\delta_{1}$ is performed for a fixed spectrum of $\hat{\mathbf{D}}$, i.e. $\delta_{1} \operatorname{Tr}(\hat{\mathbf{D}} \hat{\mathbf{H}})=\operatorname{Tr}\left(\hat{\mathbf{D}}^{\prime} \hat{\mathbf{H}}\right)-\operatorname{Tr}(\hat{\mathbf{D}} \hat{\mathbf{H}})$,

with $\hat{\mathbf{D}}^{\prime}, \hat{\mathbf{D}} \in \mathscr{M}$ and $\left\|\hat{\mathbf{D}}^{\prime}-\hat{\mathbf{D}}\right\|<\varepsilon$, with $\varepsilon$ a positive infinitesimal quantity. If $\hat{\mathbf{D}}_{0} \in \mathscr{M}$ is the solution we are looking for, then all matrices unitarily equivalent to $\hat{\mathbf{D}}_{0}$ belong also to $\mathscr{U}$ :

$$
\hat{\mathbf{D}}=\hat{\mathbf{U}} \hat{\mathbf{D}}_{0} \hat{\mathbf{U}}^{+}=\exp (-\mathrm{i} \hat{\mathbf{S}}) \hat{\mathbf{D}}_{0} \exp (\mathrm{i} \hat{\mathbf{S}})
$$

with $\hat{\mathbf{S}}$ an arbitrary hermitian operator. Inserting (2.7) into (2.6) we obtain

$$
\operatorname{Tr}\left(\left[\hat{\mathbf{D}}_{0}, \delta \hat{\mathbf{S}}\right] \hat{\mathbf{H}}\right)=\operatorname{Tr}\left(\left[\hat{\mathbf{H}}, \hat{\mathbf{D}}_{0}\right] \delta \hat{\mathbf{S}}\right)=0 .
$$

The arbitrariness of $\delta \hat{\mathbf{S}}$ leads to the stationary condition:

$$
\left[\hat{\mathbf{H}}, \hat{\mathbf{D}}_{0}\right]=0 \text {. }
$$

The states $\hat{\mathbf{D}}_{0}$ which satisfy (2.9) are called stationary since they do not evolve in time.

From the commutation relation (2.9), it follows that there is at least one complete and orthonormal set of common eigenvectors of $\hat{\mathbf{H}}$ and $\hat{\mathbf{D}}_{0}$, which we denote by $\{|m\rangle\}$ :

$$
\begin{aligned}
& \hat{\mathbf{H}}|m\rangle=E_{m}|m\rangle \quad E_{1} \leqslant E_{2} \leqslant E_{3} \leqslant \ldots \\
& \hat{\mathbf{D}}_{0}|m\rangle=P_{m}|m\rangle .
\end{aligned}
$$

In order to ensure that the extremum thus found is a minimum, we require furthermore that

$$
\beta \operatorname{Tr}(\hat{\mathbf{D}} \hat{\mathbf{H}}) \geqslant \beta \operatorname{Tr}\left(\hat{\mathbf{D}}_{0} \hat{\mathbf{H}}\right)
$$

for all $\hat{\mathbf{D}} \in \mathscr{K}$. This inequality may be written, up to second order in $\hat{\mathbf{S}}$, as

$$
-\frac{1}{2} \beta \operatorname{Tr}\left(\left[\hat{\mathbf{S}},\left[\hat{\mathbf{S}}, \hat{\mathbf{D}}_{0}\right]\right] \hat{\mathbf{H}}\right) \geqslant 0 .
$$

The trace is easily evaluated, using the basis of $(2.10)$, yielding

$$
\begin{aligned}
& \frac{1}{2} \beta\left\{\operatorname{Tr}\left(\hat{\mathbf{S}} \hat{\mathbf{D}}_{0} \hat{\mathbf{S}} \hat{\mathbf{H}}\right)-\operatorname{Tr}\left(\hat{\mathbf{S}}^{2} \hat{\mathbf{D}}_{0} \hat{\mathbf{H}}\right)\right\} \\
& \quad=\frac{1}{2} \beta \sum_{m n}\left|S_{m n}\right|^{2}\left(P_{n}-P_{m}\right) E_{m} \\
& \quad=\frac{1}{4} \beta \sum_{m n}\left|S_{m n}\right|^{2}\left(P_{n}-P_{m}\right)\left(E_{m}-E_{n}\right) \geqslant 0
\end{aligned}
$$

with $S_{m n}=\langle m|\hat{\mathbf{S}}| n\rangle$.

The inequality (2.13) is fulfilled in one of the following cases:

$$
\beta>0 \text { and }\left(P_{n}-P_{m}\right)\left(E_{m}-E_{n}\right) \geqslant 0
$$

or

$$
\beta<0 \text { and }\left(P_{n}-P_{m}\right)\left(E_{m}-E_{n}\right) \leqslant 0 .
$$

The first case means that higher $P_{m}$ values correspond to lower energies $E_{m}$. The second case, with higher $P_{m}$ corresponding to higher energies $E_{m}$, may only occur if the energy spectrum is bounded from above, as a consequence of the normalisation condition (2.1). We can therefore have equilibrium (stationarity) at negative temperatures in such physical systems (see e.g. Landsberg 1978b). It is well known that nuclear and atomic spin systems can be prepared in negative temperature states.

The states, defined by (2.9) and (2.14), were called 
'passive states' by Pusz and Woronovicz (1978) (see also Lenard 1978). A system prepared in some passive state has the property of being able to receive energy from the exterior. In fact, on the basis of condition (2.14) it has been proved (Pusz and Woronovicz 1978, Lenard 1978) that if an external perturbation, described by an hermitian time-dependent operator, is activated during a restricted amount of time in a finite quantal system, then the system gains energy from the outside. The name 'passive states' is suggestive, since a thermally isolated system prepared in such a state is ready to accept energy from the surroundings, if the external conditions are changed. Work is therefore done on the system and not $b y$ the system. On the contrary, states defined by (2.9) and (2.15) do not have this property, being ready to give energy away, under modification of the external conditions.

(ii) Minimisation with respect to the eigenvalues of $\mathbf{D}$ In this stage we keep fixed the eigenvectors, which have been found in stage (i), and vary with respect to the eigenvalues. We are therefore searching for a minimum of $F[\hat{\mathbf{D}}]$ in the family of passive states $\hat{\mathbf{D}}_{0}=\hat{\mathbf{D}}_{0}\left[\left\{P_{m}\right\}\right]=\Sigma_{m}|m\rangle P_{m}\langle m|$, each one belonging to a different set $\mathscr{H}=\left\{\hat{\mathbf{D}}_{0}\right.$ : spectrum of $\left.\hat{\mathbf{D}}_{0}=\left\{\boldsymbol{P}_{m}\right\}\right\}$. We express the variational problem by

$$
\delta_{2} F\left[\hat{\mathbf{D}}_{0}\right]=0,
$$

where $\delta_{2}$ denotes a variation with respect to the $P_{m}$, i.e. $\delta_{2} F\left[\hat{\mathbf{D}}_{0}\right]=F\left[\hat{\mathbf{D}}_{0}^{\prime}\right]-F\left[\hat{\mathbf{D}}_{0}\right]$, with $\left(\hat{\mathbf{D}}_{0}^{\prime}-P_{m}^{\prime}\right)|m\rangle=\left(\hat{\mathbf{D}}_{0}-\right.$ $\left.P_{m}\right)|m\rangle=0, m=1,2, \ldots$, and $\left\|\hat{\mathbf{D}}_{0}^{\prime}-\hat{\mathbf{D}}_{0}\right\|<\varepsilon$.

Using the common eigenvectors of $\hat{\mathbf{D}}_{0}$ and $\hat{\mathrm{H}}$, the functional (2.4) may be written as

$$
F\left[\hat{\mathbf{D}}_{0}\right]=\sum_{m}\left(P_{m} \log P_{m}+\beta P_{m} E_{m}\right) .
$$

Incorporating the normalisation (2.1) as a subsidiary condition, associated with the Lagrange multiplier $\alpha$, the variational problem is expressed by

$$
\frac{\partial}{\partial P_{m}}\left(F\left[\left\{P_{m}\right\}\right]-\alpha \sum_{m} P_{m}\right)=0
$$

leading to

$$
P_{m}=\exp (\alpha-1) \exp \left(-\beta E_{m}\right)
$$

It is straightforward to verify that the Lagrange multiplier $\alpha$ is given by

$$
\alpha=1-\log \sum_{m} \exp \left(-\beta E_{m}\right)=1-\log Z
$$

so that $(2.19)$ reads

$$
P_{m}=Z^{-1} \exp \left(-\beta E_{m}\right)
$$

where

$$
Z=\sum_{m} \exp \left(-\beta E_{m}\right)
$$

These values are always positive, as required by the positive definiteness of $\hat{D}$, and satisfy the requirements
(2.14) or (2,15). The second derivative of (2.17) yields

$$
\frac{\partial^{2}}{\partial P_{m}^{2}}\left(F\left[\left\{P_{m}\right\}\right]-\alpha \sum_{m} P_{m}\right)=\frac{1}{P_{m}} \geqslant 0
$$

ensuring the stability of the minimum. It may easily be verified that (2.21) corresponds to an absolute minimum of (2.4).

The set of eigenvalues (2.21) characterise the state of thermal equilibrium:

$$
\hat{\mathbf{D}}_{0, \text { can }}=\sum_{m}|m\rangle Z^{-1} \exp \left(-\beta E_{m}\right)<m \mid \text {. }
$$

Note that the variational procedure (ii) has defined a mapping (2.21) between the eigenvalues of $\hat{D}$ and the eigenvalues of $\hat{H}$. If the eigenvalues of $\hat{H}$ are degenerate, the stage (i) alone does not necessarily ensure that $\hat{\mathbf{D}}=f(\hat{\mathbf{H}})$, with $f$ a non-increasing functional (we may have $P_{m} \neq P_{n}$, while $E_{m}=E_{n}$ ), but the whole variational method implies the existence of such a functional relationship, which is called 'structural stability' by Lenard (1978).

In quantum statistical mechanics, ergodicity is related to the non-degeneracy of the energy spectrum (Mello and Moshinsky 1972, Bongaarts and Siskens 1973, 1974), since, in contrast to integrable systems, ergodic systems do not admit other invariants besides the Hamiltonian. For an ergodic quantal system, the eigenvalues of the density matrix associated with a passive state must decrease with the eigenenergies as indicated by the strict inequality in (2.14).

We emphasise that the exact form of the functional relationship between $\hat{D}$ and $\hat{H}$ is a consequence of the definition adopted for information. The canonical density matrix (2.23) follows from the two-step process of minimisation, so that

$F\left[\hat{\mathbf{D}}_{\text {O.can }}\right]=-\log Z \leqslant \operatorname{Tr}(\hat{\mathbf{D}} \log \hat{\mathbf{D}})+\beta \operatorname{Tr}(\hat{\mathbf{D}} \hat{\mathbf{H}})$,

the equality holding if and only if $\hat{\mathbf{D}}=\hat{\mathbf{D}}_{0 \text {. san }}$.

\section{Particular results}

We are now free to insert in the right-hand side (RHS) of (2.24) any trial density matrix, obtaining in this way particular inequalities.

\subsection{Inequality between the arithmetic and geometric means}

This inequality is achieved if the eigenvectors of the density matrix are optimised but the eigenvalues are not. Let us consider a Hamiltonian bounded from above (this property is realistic for a spin system), which has eigenvectors $|m\rangle, m=1, \ldots, N$. We insert

$$
\hat{\mathbf{D}}=\sum_{m=1}^{N}|m\rangle N^{-1}\langle m|
$$


in the RHS of (2.24), obtaining

$$
\begin{gathered}
-\log \sum_{m=1}^{N} \exp \left(-\beta E_{m}\right) \leqslant(\beta / N) \sum_{m=1}^{N} E_{m}-\log N \\
\sum_{m=1}^{N} \exp \left(-\beta E_{m}\right) \geqslant \exp \left(-(\beta / N) \sum_{m=1}^{N} E_{m}+\log N\right) \\
N^{-1} \sum_{m=1}^{N} a_{m} \geqslant\left(\prod_{m=1}^{N} a_{m}\right)^{1 / N}
\end{gathered}
$$

with $a_{m}=\exp \left(-\beta E_{m}\right) \geqslant 0$. Equality in (3.2) occurs if and only if there is full degeneracy of the spectrum of $\hat{\mathrm{H}}: Z^{-1} \exp \left(-\beta E_{m}\right)=N^{-1}$.

The inequality (3.2) is the celebrated inequality between the arithmetic and the geometric means, which is the cornerstone of the mathematical theory of inequalities (Bellman and Beckenbach 1971). We have derived it from the basic postulates of quantum statistical mechanics in a vein very similar to the thermodynamic proof of the same inequality proposed by Landsberg (Landsberg 1978a).

The inequality between the arithmetic and the geometric means may be seen as a very particular case of a complete chain of inequalities between 'generalised means' (Landsberg 1980a, b).

The method we have presented may also allow generalisations of inequality (3.2). For that purpose, let us consider an ansatz for $\hat{\mathbf{D}}$, which is less restrictive than (3.1): $\hat{\mathbf{D}}=\Sigma_{m}|m\rangle P_{m}\langle m|$, with the $P_{m}$ positive real numbers such that $\Sigma_{m} P_{m}=1$. Inserting this density matrix in (2.24) we obtain a generalisation of the inequality between the arithmetic and the geometric means:

$$
\begin{aligned}
& \sum_{m} \exp \left(-\beta E_{m}\right) \\
& \geqslant \exp \left(\sum_{m}\left[-\log \left(P_{m}\right)^{P_{m}}-\beta P_{m} E_{m}\right]\right) \\
& \sum_{m} \exp \left(-\beta E_{m}\right) \geqslant \prod_{m}\left(\exp \left(-\beta E_{m}\right) / P_{m}\right)^{P_{m}}
\end{aligned}
$$

or, with $X_{m}=\exp \left(-\beta E_{m}\right) / P_{m} \geqslant 0$,

$$
\sum_{m} X_{m} P_{m} \geqslant \prod_{m} X_{m}^{P_{m}}
$$

This is one of the inequalities betwen generalised means discussed by Landsberg (1980a, b).

A further extension of our method, in order to achieve other inequalities, would consist in measuring the information with a functional different from (2.3). Care should then be taken when establishing links with physical reality, since these choices are not compatible with all the properties of entropy.

\subsection{Peierls inequality}

To deduce the Peierls inequality we consider the case where the eigenvectors of $\hat{D}$ are not optimised but the eigenvalues are, i.e. we suppose that step (ii) is the only one to be performed.

We assume therefore as an ansatz for D̂

$$
\hat{\mathbf{D}}=\sum_{m}|\tilde{m}\rangle \tilde{P}_{m}\langle\tilde{m}|
$$

where

$$
\tilde{P}_{m}=\exp (-\beta\langle\tilde{m}|\hat{\mathbf{H}}| \tilde{m}\rangle) / \sum_{m} \exp (-\beta\langle\tilde{m}|\hat{\mathbf{H}}| \tilde{m}\rangle)
$$

with $\{|\bar{m}\rangle\}$ a set of orthonormal vectors, which does not need to be complete. From (2.24) it follows that

$$
-\log Z \leqslant-\log \sum_{m} \exp (-\beta\langle\tilde{m}|\hat{\mathbf{H}}| \tilde{m}\rangle)
$$

or further

$$
Z \geqslant \sum_{m} \exp (-\beta\langle\tilde{m}|\hat{\mathbf{H}}| \tilde{m})) .
$$

Equality holds if and only if the trial set coincides with the set of eigenvectors of the Hamiltonian, being therefore complete, i.e. $\{|\tilde{m}\rangle\} \equiv\{|m\rangle\}$.

The inequality (3.9) expresses the Peierls theorem (Peierls 1938). Various proofs appear in the literature (e.g. Huang 1963, Falk 1963, Huber 1970) but the present one seems to be simpler. Moreover, the canonical density matrix is here not assumed to be known as in those references, but is derived from first principles.

\subsection{Bogolyubov inequality}

Let us give an example of a trial density matrix that obeys neither the prescriptions indicated in (i) nor in (ii), but may be useful as an approximative tool.

For that purpose, we split the Hamiltonian into two parts $\hat{H}_{1}$ and $\hat{H}_{2}$

$$
\hat{\mathbf{H}}=\hat{\mathbf{H}}_{1}+\hat{\mathbf{H}}_{2} \text {. }
$$

We asume the solution of the diagonalisation problem for $\hat{\mathbf{H}}_{1}$ is known, while $\hat{\boldsymbol{H}}_{2}$ is to be considered as a perturbation. Let us choose for $\hat{\mathbf{D}}$ the canonical density matrix, $\hat{\mathbf{D}}_{1}$, associated with the Hamiltonian $\hat{\mathbf{H}}_{1}$.

$$
\hat{\mathbf{D}}_{1}=\exp \left(-\beta \hat{\mathbf{H}}_{1}\right) / \operatorname{Tr} \exp \left(-\beta \hat{\mathbf{H}}_{1}\right)
$$

Then, inequality (2.24) becomes

$$
-\log Z \leqslant F_{1}\left[\hat{\mathbf{D}}_{1}\right]+\beta \operatorname{Tr}\left(\hat{\mathbf{D}}_{1} \hat{\mathbf{H}}_{2}\right)
$$

with

$$
F_{1}\left[\hat{\mathbf{D}}_{\mathrm{l}}\right]=\operatorname{Tr}\left(\hat{\mathbf{D}}_{\mathrm{l}} \log \hat{\mathbf{D}}_{1}\right)+\beta \operatorname{Tr}\left(\hat{\mathbf{D}}_{1} \hat{\mathbf{H}}_{1}\right) .
$$

The inequation (3.11) is referred to as the Bogolyubov inequality or Peierls-Bogolyubov inequality, since it has been attributed to N N Bogolyubov by Kvasnikov (1956). The demonstration we have presented avoids the perturbative treatment of Feynman (Feynman 1972).

From the variational procedure, it follows that the Peierls inequality is superior to the Bogolyubov one. If we have at our disposal the eigenvectors of $\hat{\mathbf{H}}_{1}$, which 
we denote by $\left|m_{1}\right\rangle$, the lowest upper bound for $F\left[\hat{\mathbf{D}}_{0 \text {, can }}\right]$ is provided by the Peierls theorem:

$$
\begin{aligned}
-\log Z \leqslant-\log \sum_{m_{1}} \exp \left(-\beta\left\langle m_{1}|\hat{\mathbf{H}}| m_{1}\right\rangle\right) \\
\leqslant F_{1}\left[\hat{\mathbf{D}}_{1}\right]+\beta \operatorname{Tr}\left(\hat{\mathbf{D}}_{1} \hat{\mathbf{H}}_{2}\right)
\end{aligned}
$$

\section{Stable and metastable equilibrium}

We emphasise that thermal equilibrium states are passive states but that the opposite is not necessarily true. Passive states may be considered as representing a situation of equilibrium different from the stable thermal equilibrium. We prefer to call them states of metastable equilibrium, for reasons which may become clear from the following arguments.

In classical thermodynamics, metastable states are defined as states which exist during a time interval long enough for them to be considered macroscopic states, having measurable properties like energy, entropy, etc. As a result of some small perturbation, they decay irreversibly into states of thermal equilibrium, which have lower Helmholtz free energy.

The passive states are equilibrium states in the sense that they are stable against any unitary transformation. A system prepared in an equilibrium state does not evolve in time, if the dynamical evolution is described by the Liouville-von Neumann equation

$$
\dot{\hat{\mathbf{D}}}=-\mathrm{i}[\hat{\mathrm{H}}, \hat{\mathrm{D}}]
$$

where $\hat{\mathbf{H}}$ is the Hamiltonian containing the interactions within the given system. It is however known that the entropy is a constant of motion for a system governed by equation $(4.1)$. If one wishes to account for the increase of entropy required by the second law of thermodynamics, one is left with two alternatives: (1) to modify the definition of entropy (this is the reason for introducing the 'coarse-grained' entropy) or (2) to accept that the dynamics are not completely described in equation (4.1) (the master equation is meaningful in this context).

In non-isolated systems, external fluctuations are responsible for the evolution towards thermal equilibrium. These residual interactions with the surroundings (a heat bath, for instance), which are not contained in $\hat{\mathbf{H}}$, will lead from the neighbourhood of a passive state to the state of thermal equilibrium. The first type of equilibrium is therefore short-term or metastable, while the second is long-term or stable.

It should be pointed out that the machinery of linear response against external known fields only requires the existence of a passive state, so that one may consider oscillations (with real frequencies and described by well defined boson operators) around metastable or stable states. The persistence of oscillations around metastable states depends on the comparison of the period with the time of relaxation towards thermal equilibrium. If the period is much smaller than the relaxation time, we may consider quasi-stationary mixed states oscillating around a stationary solution.

Recently, the question of excitation around thermal equilibrium has been investigated in nuclear and solid state physics. Evidence has been found for dipolar motion in 'compound nuclei', while modern neutronscattering experiments have revealed the persistence of spin-waves up to the Curie temperature. We refer the reader to the literature for a detailed theoretical treatment of these phenomena (Providência and Fiolhais, 1985a, b, Brajczewska et al 1986).

\section{Conclusions}

We have proposed a pedagogical approach to the theory of the canonical density matrix, consisting of a double minimisation of the free energy with respect to (i) the eigenvectors and (ii) the eigenvalues of trial density matrices.

With the assumption of equal eigenvalues for the trial density matrix, minimisation (i) led to the inequality between the arithmetic and the geometrical means, which may be seen as a member of a chain of inequalities between generalised means. On the other hand, the Peierls theorem has been demonstrated on the basis of the minimisation (ii) within the family of all density matrices which admit as eigenvectors a given orthonormal set. The Bogolyubov inequality has been obtained with an ansatz for $\hat{D}$ which corresponds to the minimisations indicated in (i) and (ii), taking some approximation to the true Hamiltonian.

The inequality between the arithmetic and the geometric means is an example of the intimate connection between the principles of physics and mathematics, although one should agree with Landsberg (1980a) that the physical proofs such as the one we have presented are not necessarily more convincing or simpler than the standard ones.

Passive states have been characterised by their stability against canonical transformations and their ability to receive energy from the outside, when the external conditions are changed. Thermal equilibrium states have been defined by a certain functional dependence of the density matrix on the Hamiltonian, being globally stable. Oscillating states may exist around any passive state, not necessarily states of thermal equilibrium.

We comment, finally, that for classical systems the density matrix should be replaced by a distribution function in phase space. Then, concepts like eigenvalues and eigenvectors are lost, so that the Peierls theorem does not have a classical translation. In contrast, the Bogolyubov inequality does have a classical counterpart (Wehrl 1978).

\section{Acknowledgments}

This work has been partially supported by the Instituto Nacional de Investigação Científica and by the Calouste 
Gulbenkian Foundation. We are indebted to Sir R Peierls for some useful comments and criticism, which have stimulated the writing of this article.

\section{References}

Bellman R and Beckenbach E F 1971 Inequalities (Berlin: Springer)

Brajczewska M, Fiolhais C and Providência J da 1986 Physica A 137196

Bongaarts P J and Siskens T J 1973 Physica 68314 1974 Physica 71529

Falk H 1963 Physica 291114

Feynman R P 1972 Statistical Mechanics (Reading. MA: Benjamin)

Huang K 1963 Statistical Mechanics (New York: Wiley)

Huber A 1970 Methods and Problems of Theoretical Phisics - in honour of $R$ Peierls ed. J E Bowcock (Amsterdam: North Holland)
Katz A 1967 Principles of Statistical Mechanics (San Francisco: Freeman)

Kvasnikov I A 1956 Dokl. Akad. Nauk. SSSR 110 755

Landsberg P T 1978a Phys. Lett. A67 1

1978b Thermodvnamics and Statistical Mechanics

(Oxford: Oxford University Press)

1980a Phys. Lett. A78 29

1980b J. Math. Anal. Appl. 76209

Lenard A 1978 J. Stat. Phys. 19575

Mello P and Moshinsky M 1972 Ann. Phys. 741

Peierls R 1938 Phi's. Rev. 54918

Providência J da and Fiolhais C 1985a Nucl. Phy's. A 435 190

1985b Portugaliae Phisica 1169

Pusz W and Woronovicz S L 1978 Commun. Math. Phis. 58273

Wehrl A 1978 Rev. Mod. Phys. 50221 